\title{
Secure Two-Party Computation via Cut-and-Choose Oblivious Transfer
}

\author{
Yehuda Lindell` and Benny Pinkas ${ }^{\star \star}$ \\ Dept. of Computer Science, Bar-Ilan University, Israel
}

\begin{abstract}
Protocols for secure two-party computation enable a pair of parties to compute a function of their inputs while preserving security properties such as privacy, correctness and independence of inputs. Recently, a number of protocols have been proposed for the efficient construction of two-party computation secure in the presence of malicious adversaries (where security is proven under the standard simulationbased ideal/real model paradigm for defining security). In this paper, we present a protocol for this task that follows the methodology of using cut-and-choose to boost Yao's protocol to be secure in the presence of malicious adversaries. Relying on specific assumptions (DDH), we construct a protocol that is significantly more efficient and far simpler than the protocol of Lindell and Pinkas (Eurocrypt 2007) that follows the same methodology. We provide an exact, concrete analysis of the efficiency of our scheme and demonstrate that (at least for not very small circuits) our protocol is more efficient than any other known today.
\end{abstract}

\section{Introduction}

\subsection{Background}

Protocols for secure two-party computation enable a pair of parties $P_{1}$ and $P_{2}$ with private inputs $x$ and $y$, respectively, to compute a function $f$ of their inputs while preserving a number of security properties. The most central of these properties are privacy (meaning that the parties learn the output $f(x, y)$ but nothing else), correctness (meaning that the output received is indeed $f(x, y)$ and not something else), and independence of inputs (meaning that neither party can choose its input as a function of the other party's input). The standard way of formalizing these security properties is to compare the output of a real protocol execution to an "ideal execution" in which the parties send their inputs to an incorruptible trusted party who computes the output for the parties. Informally speaking, a protocol is then secure if no real adversary attacking the real protocol can do more harm than an ideal adversary (or simulator) who interacts in the ideal model [1314/2623]. An important parameter when considering this

\footnotetext{
* lindell@cs.biu.ac.il.Research supported by the European Research Council as part of the ERC project LAST, and by the ISRAEL SCIENCE FOUNDATION (781/07).

** benny@pinkas.net Research supported by the European Research Council as part of the ERC project SFEROT and by the EU project CACE.
} 
problem relates to the power of the adversary. The two most studied models are the semi-honest model (where the adversary follows the protocol specification exactly but tries to learn more than it should by inspecting the protocol transcript) and the malicious model (where the adversary can follow any arbitrary polynomial-time strategy).

In the 1980s powerful feasibility results were proven, showing that any probabilistic polynomial-time functionality can be securely computed in the presence of semi-honest adversaries 33 and in the presence of malicious adversaries [13]. These results showed that it is possible to achieve such secure protocols, but did not demonstrate how to do so efficiently (where by efficiency we mean a protocol that can be implemented and run in practice). To be more exact, the protocol of 33 for semi-honest adversaries is efficient. However, achieving security efficiently for the case of malicious adversaries is far more difficult. In fact, until recently, no efficient general protocols were known at all, where a general protocol is one that can be used for computing any functionality.

This situation has changed in the past few years, possibly due to increasing interest from outside the cryptographic community in secure protocols that are efficient enough to be used in practice. The result has been that a number of secure two-party protocols were presented that are secure in the presence of malicious adversaries, where security is rigorously proven according to the aforementioned ideal/real model paradigm 2024 28 18. Interestingly, these protocols all take novel, different approaches and so the secure-protocol skyline is more diverse than before, providing the potential for taking the protocols a step closer to very high efficiency. These protocols are discussed in more detail in Section 1.3.

We remark that the protocol of [24] has been implemented for the non-trivial problem of securely computing the AES block cipher (pseudorandom function), where one party's input is a secret key and the other party's input is a value to be "encrypted" [31]. A Boolean circuit for computing this function was designed with approximately 33,000 gates, and the protocol of 24] was implemented for this circuit. Experiments showed that the running-time of the protocol was between 18 and 40 minutes, depending on the assumptions taken on the primitives used to implement the protocol. Although this is quite a long time, for some applications it can be reasonable. In addition, it demonstrates that it is possible to securely compute functions with large circuits, and motivates the search for finding even more efficient protocols that can widen the applicability of such computations in real-world settings.

\subsection{Our Results}

In this paper, we follow the construction paradigm of [24] and significantly simplify and improve the efficiency of their construction. The approach of [24] is to carry out a basic cut-and-choose on the garbled circuit construction of Yao [33. (we assume familiarity with Yao's protocol). That is, party $P_{1}$ constructs $s$ copies of a garbled circuit and sends them to $P_{2}$, who then asks $P_{1}$ to open half of them in order to verify that they are correctly constructed. If all of the opened circuits are indeed correct, then it is guaranteed that a majority of the unopened half 
are also correct, except with probability that is negligible in a statistical security parameter $s$. Thus, $P_{1}$ and $P_{2}$ evaluate the remaining $s / 2$ circuits, and $P_{2}$ takes the output that appears in most of the evaluated circuits. As discussed in [24, $P_{2}$ cannot abort in the case that not all of the $s / 2$ circuits evaluate to the same value, even though in such a case it knows that $P_{1}$ is cheating. The reason for this is that $P_{1}$ may construct a circuit that computes $f$ in the case that $P_{2}$ 's first bit equals 0 , and otherwise it outputs random garbage. Now, with probability $1 / 2$ this faulty circuit is not opened and so is one of the circuits to be evaluated. In this case, if $P_{2}$ would abort when it saw random garbage then $P_{1}$ would know that $P_{2}$ 's first input bit equals 1 . For this reason, $P_{2}$ takes the majority output and ignores minority values without aborting.

Although intuitively appealing, the cut-and-choose approach introduces a number of difficulties which significantly affect the efficiency of the protocol of [24]. First, since the parties need to evaluate $s / 2$ circuits rather than one, there needs to be a mechanism to ensure that they use the same input in all evaluations (the solution for this for $P_{2}$ 's inputs is easy, but for $P_{1}$ 's inputs turns out to be hard). The mechanism used in [24] required constructing and sending $s^{2} \ell$ commitments. In the implementation by [31, they set $s=160$ and $\ell=128$. Thus, the overhead due to these consistency proofs is the computation and transmission of 3,276, 800 commitments! Another problem that arises in the protocol of [24] is that a malicious $P_{1}$ can input an incorrect key into one of the oblivious transfers used for $P_{2}$ to obtain the keys associated with its input wires in the garbled circuit. For example, it can set all the keys associated with 0 for $P_{2}$ 's first input bit to be garbage, thereby making it impossible for $P_{2}$ to decrypt any circuit if its first input bit indeed equals 0 . In contrast, $P_{1}$ can make all of the other keys be correct. In this case, $P_{1}$ is able to learn $P_{2}$ 's first input bit, merely by whether $P_{2}$ obtains an output or not. The important observation is that the checks on the garbled circuit carried out by $P_{2}$ do not detect this because there is a separation between the cut-and-choose checks and the oblivious transfer. The solution to this problem in [24] requires making the circuit larger and significantly increasing the size of the inputs by replacing each input bit with the exclusive-or of multiple random input bits. Finally, the analysis of 24] yields an error of $2^{-s / 17}$. Thus, in order to obtain an error level of $2^{-40}$ the parties need to exchange 680 circuits. We remark that it has been conjectured in 31 that the true error level of the protocol is $2^{-s / 4}$; however, this has not been proven.

Our protocol. We solve the aforementioned problems in a way that is far simpler and far more efficient than in 24 . In addition, we reduce the error probability to $2^{-0.311 s}$ and thus for an error of $2^{-40}$ it suffices to send only 128 circuits. This is an important improvement because the experiments of [31] demonstrate that the bottleneck in efficiency is not the exponentiations, but rather the number of circuits and the commitments for proving consistency. Thus, in our protocol we moderately increase the number of exponentiations, while reducing the number of circuits, completely removing the commitments, and also removing the need to increase the size of the inputs. We remark that the price for these improvements is that our protocol relies heavily on the decisional Diffie-Hellman (DDH) 
assumption, while the protocol of [24] used general assumptions only. We now proceed to describe our two main techniques:

1. Our solution for ensuring consistency of $P_{1}$ 's inputs is to have $P_{1}$ determine the keys associated with its own input bits via a Diffie-Hellman pseudorandom synthesizer [27. That is, $P_{1}$ chooses values $g^{a_{1}^{0}}, g^{a_{1}^{1}}, \ldots, g^{a_{\ell}^{0}}, g^{a_{\ell}^{1}}$ and $g^{r_{1}}, \ldots, g^{r_{s}}$ and then sets the keys associated with its $i$ th input bit in the $j$ th circuit to be $g^{a_{i}^{0} \cdot r_{j}}, g^{a_{i}^{1} \cdot r_{j}}$. Given all of the $\left\{g^{a_{i}^{0}}, g^{a_{i}^{1}}, g^{r_{j}}\right\}$ values and any subset of keys of $P_{1}$ 's input generated in this way, the remaining keys associated with its input are pseudorandom by the DDH assumption. Furthermore, it is possible for $P_{1}$ to efficiently prove that it is using the same input in all circuits when the keys have this nice structure.

2. As we have described, the reason that the inputs and circuits were needed to be made larger in [24] is due to the fact that the cut-and-choose circuit checks were separated from the oblivious transfer. In order to solve this problem, we introduce a new primitive called cut-and-choose oblivious transfer. This is an ordinary oblivious transfer with the sender inputting many pairs $\left(x_{1}^{0}, x_{1}^{1}\right), \ldots,\left(x_{s}^{0}, x_{s}^{1}\right)$, and the receiver inputting bits $\sigma_{1}, \ldots, \sigma_{s}$. However, the receiver also inputs a set $\mathcal{J} \subset[s]$ of size exactly $s / 2$. Then, the receiver obtains $x_{i}^{\sigma_{i}}$ for every $i$ (as in a regular oblivious transfer) along with both values $\left(x_{j}^{0}, x_{j}^{1}\right)$ for every $j \in \mathcal{J}$. The use of this primitive in our protocol intertwines the oblivious transfer and the circuit checks and solves the aforementioned problem. We also show how to implement this primitive in a highly efficient way, under the DDH assumption. We believe that this primitive is of independent interest, and could be useful in many cut-and-choose scenarios.

Efficiency analysis. Our entire protocol, including all subprotocols, is explicitly written and analyzed in a concrete and exact way for efficiency. Considerable effort has been made to optimize the constructions and reduce the constants throughout. We believe that this is of great importance when the focus of a result is efficiency. See Section 1.3 for a summary of the exact complexity of our protocol, and Section 3 for a complete analysis, with optimizations in Section 3.3.

Variants. Another advantage of our protocol over that of [24] is that we obtain a universally composable [4] variant that is only slightly less efficient than the stand-alone version. This is because our simulator only rewinds during zeroknowledge protocols. These protocols are also $\Sigma$ protocols and so can be efficiently transformed into universally composable zero-knowledge. As with our basic protocol, we provide an explicit description of this transformation and analyze its exact efficiency. Finally, we also show how our protocol yields a more efficient construction for security in the presence of covert adversaries [1], when high values of the deterrent factor $\epsilon$ are desired.

\subsection{Comparison to Other Protocols}

We provide an analysis of the efficiency of recent protocols for secure two-party computation. Each protocol takes a different approach, and thus the approaches 
may yield more efficient instantiations in the future. Nevertheless, as we will show, our protocol is significantly more efficient than the current best instantiations of the other approaches (at least, for not very small circuits).

- Committed input method (Jarecki-Shmatikov [20]): The secure twoparty protocol of [20] works by constructing a single circuit and proving that it is correct. The novelty of this protocol is that this can be done with only a constant number of (large modulus) exponentiations per gate of the circuit. Thus, for circuits that are relatively small, this can be very efficient. However, an exact count gives that approximately 720 exponentiations are required per gate. Thus, even for small circuits, this protocol is not yet practical. For large circuits like AES with 33,000 gates, the number of exponentiations is very large $(23,760,000$ for AES), and is not realistic. (The authors comment that if efficient batch proofs can be found for the languages they require then this can be significantly improved. However, to the best of our knowledge, no such improvements have yet been made. Furthermore, for a large circuit we believe it unlikely that this method will yield a highly efficient protocol).

- LEGO (Nielsen-Orlandi [28]): The LEGO protocol 28] follows the cutand-choose methodology in a completely different way. Specifically, the circuit constructor first sends the receiver many gates, and the receiver checks that they are correctly constructed by asking for some to be opened. After this stage, the parties interact in a way that enables the gates to be securely soldered (like Lego blocks) into a correct circuit. Since it is not guaranteed that all of the gates are correct, but just a vast majority, a fault tolerant circuit of size $O(s \cdot|C| / \log |C|)$ is constructed, where $s$ is a statistical security parameter. The error as a function of $s$ is $2^{-s}$ and the constant inside the "O" notation for the number of exponentiations is 32 [29]. Thus, for an error of $2^{-40}$ we have that the overall number of exponentiations carried out by the parties is $1280 \cdot|C| / \log |C|$. For large circuits, like that of AES, this is unlikely to be practical. (For example, for the AES circuit with 33,000 gates we have that the parties need to carry out 2,816,000 exponentiations. Observe that due to the size of the circuit, the $\log |C|$ factor is significant in making the protocol more efficient than [20, as predicted in 28. This protocol also relies on the DDH assumption. It is worthy to note that exponentiations in this protocol are in a regular "Diffie-Hellman" group and so Elliptic curves can be used, in contrast to [20] who work in $\mathbb{Z}_{N}^{*}$ ).

- Virtual multiparty method (Ishai et al. [18,19]): This method works by having the parties simulate a virtual multiparty protocol with an honest majority. The cost of the protocol essentially consists of the cost of running a semi-honest protocol for computing the multiplication of additive shares, for every multiplication carried out by a party in a multiparty protocol with honest majority. Thus, the actual efficiency of the protocol depends heavily on the multiparty protocol to be simulated, and the semi-honest protocols used for simulating the multiparty protocol. An asymptotic analysis demonstrates that this method may be competitive. However, no concrete analysis has been carried out, and it is currently an open question whether or not it 
is possible to instantiate this protocol in a way that will be competitive with other known protocols.

- Cut-and-choose on circuits (Lindell-Pinkas [24]): Since this protocol has been discussed at length above, we just briefly recall that the complexity of the protocol is $O(\ell)$ oblivious transfers for input-length $\ell$ (where the constant inside here is not small because of the need to increase the number of $P_{2}$ 's inputs), and the construction and computation of $s$ garbled circuits and of $s^{2} \ell$ commitments. In addition, the proven error of the protocol is $2^{-s / 17}$ and its conjectured error is $2^{-s / 4}$. The actual value has a significant impact on the efficiency.

In contrast to the above, the complexity of our protocol is as follows. The parties need to compute $15 s \ell+39 \ell+10 s+6$ exponentiations, where $\ell$ is the input length and $s$ is a statistical security parameter discussed below. We further show that with optimizations the $15 \mathrm{~s} \ell$ component can be brought down to just $\mathbf{5 . 6 6 s} \boldsymbol{\ell}$ full exponentiations, and if preprocessing can be used then only $s \ell / 2$ full exponentiations need to be computed after the inputs become known. In addition, the protocol requires the exchange of $7 s \ell+22 \ell+7 s+5$ group elements, and has 12 rounds of communication. Finally, there are $6.5|C| s$ symmetric encryptions for constructing and decrypting the garbled circuits and $4|C| s$ ciphertexts sent for transmitting these circuits. An important factor here is the value of $s$ needed. The error of our protocol is $\mathbf{2}^{-\mathbf{0 . 3 1 1} s}$ and so for an error of $2^{-40}$ it suffices to set $s=\mathbf{1 2 8}$. (The overhead of computing an AES circuit, after preprocessing, with $|C|=33,000$, and $s=\ell=128$, is therefore about 93,000 exponentiations, 27,500,000 symmetric encryptions, and communicating 28.6 Mbytes, where about $95 \%$ of the communication is spent on sending the garbled circuits). Finally, we stress also that all of our exponentiations are of the basic Diffie-Hellman type and so can be implemented over Elliptic curves, which is much cheaper than RSA-type operations.

Full version. In this extended abstract we do not have space to present the proofs of security of our protocols. A full version of this paper appears in the Cryptology ePrint Archive (report 2010/284).

\section{Cut-and-Choose Oblivious Transfer}

\subsection{The Functionality and Construction Overview}

Our protocol for secure two-party computation uses a new primitive that we call cut-and-choose oblivious transfer. Loosely speaking, a cut-and-choose OT is a batch oblivious transfer protocol (meaning an oblivious transfer for multiple pairs of inputs) with the additional property that the receiver can choose a subset of the pairs (of a predetermined size) for which it learns both values. This is a very natural primitive which has clear applications for protocols that are based on cut-and-choose, as is our protocol here for general two-party computation. 
The cut-and-choose OT functionality, denoted $\mathcal{F}_{\text {ccot }}$, with parameter $s$, is formally defined in Figure 1, together with a variant functionality that we will need, which considers the case that $R$ is forced to use the same choice $\sigma$ in every transfer. This variant is denoted $\mathcal{F}_{\text {ccot }}^{S}$.

\section{FIGURE 1 (The cut-and-choose OT functionalities)}

The cut-and-choose OT functionality $\mathcal{F}_{\text {ccot }}$ :

- Inputs:

- $S$ inputs a vector of pairs $\bar{x}=\left\{\left(x_{0}^{i}, x_{1}^{i}\right)\right\}_{i=1}^{s}$

- $R$ inputs $\sigma_{1}, \ldots, \sigma_{s} \in\{0,1\}$ and a set of indices $\mathcal{J} \subset[s]$ of size exactly $s / 2$.

- Output: If $\mathcal{J}$ is not of size $s / 2$ then $S$ and $R$ receive $\perp$ as output. Otherwise,

- For every $j \in \mathcal{J}$ the receiver $R$ obtains the pair $\left(x_{0}^{j}, x_{1}^{j}\right)$.

- For every $j \notin \mathcal{J}$ the receiver $R$ obtains $x_{\sigma_{j}}^{j}$.

\section{The single-choice cut-and-choose OT functionality $\mathcal{F}_{\text {ccot }}^{S}$ :}

- Inputs: The same as above, but with $R$ having only a single input bit $\sigma$.

- Output: As above, but with $R$ obtaining the value $x_{\sigma}^{j}$ for every $j \notin \mathcal{J}$.

In order to motivate the usefulness of this functionality, we describe its use in our protocol. Oblivious transfer is used in Yao's protocol so that the party computing the garbled circuit (call it $P_{2}$ ) can obtain the keys (garbled values) on the wires corresponding with its input while keeping its input secret. It is crucial that $P_{2}$ obtain only a single key for each wire, since this is what ensures that it can only obtain a single output. When applying cut-and-choose, many circuits are constructed and then half of them are opened, where opening means that $P_{2}$ receives all of the input keys to the circuit, enabling it to decrypt all garbled gates and check that they were correctly constructed. By using cut-andchoose OT, $P_{2}$ receives all of its keys in the circuits to be opened directly, in contrast to having $P_{1}$ send them separately after the indices of the circuits to be opened are sent from $P_{2}$ to $P_{1}$. The advantage of this approach is that $P_{1}$ cannot use different keys in the OT and when opening the circuit. See Section 3.1 for discussion on why this is important.

In cut-and-choose on Yao's protocol, one oblivious transfer is needed for every bit of $P_{2}$ 's input (equivalently, every wire on the circuit), and $P_{2}$ should receive the keys associated with this fixed bit in all of the circuits. In order to ensure that $P_{2}$ uses the same input in all circuits, we devised a single-choice variant of cut-and-choose OT. In the full version, we separately present the basic variant since it is of independent interest and may be useful in other applications.

\subsection{Constructing a Single-Choice Cut-and-Choose OT Protocol}

The starting point for our construction of cut-and-choose OT is the universally composable protocol of Peikert et al. 30; we refer only to the instantiation of 
their protocol based on the DDH assumption because this is the most efficient. However, our protocol can use any of their instantiations. The protocol of [30] is cast in the common reference string (CRS) model, where the CRS is a tuple $\left(g_{0}, g_{1}, h_{0}, h_{1}\right)$ where $g_{0}$ is a generator of a group of order $q$ (in which DDH is assumed to be hard), $g_{1}=\left(g_{0}\right)^{y}$ for some random $y$, and it holds that $h_{0}=\left(g_{0}\right)^{a}$ and $h_{1}=\left(g_{1}\right)^{b}$ where $a \neq b$. We first observe that it is possible for the receiver to choose this tuple itself, as long as it proves that it indeed fulfills the property that $a \neq b$. Furthermore, this can be proven very efficiently by setting $b=a+1$; in this case, the proof that $b=a+1$ is equivalent to proving that $\left(g_{0}, g_{1}, h_{0}, \frac{h_{1}}{g_{1}}\right)$ is a Diffie-Hellman tuple (note that the security of [30] is based only on $a \neq b$ and not on these values being independent of each other). We thus obtain a highly efficient version of the protocol of 30 in the stand-alone model.

Next, observe that the protocol of [30] has the property that if $\left(g_{0}, g_{1}, h_{0}, h_{1}\right)$ is a Diffie-Hellman tuple (i.e., if $a=b$ ) then it is possible for the receiver to learn both values (of course, in a real execution this cannot happen because the receiver proves that $a \neq b$ ). This property is utilized by 30. to prove universal composability; in their case the simulator can choose the CRS so that $a=b$ and then learn both inputs of the sender, something that is needed for proving simulation-based security. However, in our case, we want the receiver to be able to sometimes learn both inputs of the sender. We can therefore utilize this exact property and have the receiver choose $s$ pairs $\left\{\left(h_{0}^{j}, h_{1}^{j}\right)\right\}_{j=1}^{s}$ such that for $s / 2$ of the pairs $\left(h_{0}^{j}, h_{1}^{j}\right)$ it holds that $a \neq b$ (ensuring that it learns only one input) and for $s / 2$ of the pairs $\left(h_{0}^{j}, h_{1}^{j}\right)$ it holds that $a=b$ (enabling it to learn both inputs by actually running the UC simulator). This therefore provides the exact cut-and-choose property in the OT that is needed. Of course, the receiver must also prove that it behaved in this way. Specifically, it proves in zero-knowledge that $s / 2$ out of $s$ pairs are such that $a \neq b$. This proof too can be computed at low cost using the technique of Cramer et al. [7; see the full version of the paper for a full description and efficiency analysis of the zero-knowledge protocol.

In the oblivious transfer protocol, the receiver with an input $\sigma$ chooses a random $r$ and sends $\left(g_{\sigma}\right)^{r}$ and $\left(h_{\sigma}^{1}\right)^{r}, \ldots,\left(h_{\sigma}^{s}\right)^{r}$ to the sender, using the $g_{0}, g_{1},\left(h_{0}^{j}, h_{1}^{j}\right)$ values sent previously. In order to ensure that the single-choice property holds, the receiver $R$ must prove that it used the same $\sigma$ in every computation of $\left(h_{\sigma}^{j}\right)^{r}$. The protocol has been carefully designed so that the way that $R$ chooses the values enables this proof to be carried out efficiently. Specifically, the required zero-knowledge proof is that there exists an $r \in \mathbb{Z}_{q}$ such that either $g^{\prime}=\left(g_{0}\right)^{r}$ and $h_{j}=\left(h_{0}^{j}\right)^{r}$ for every $1 \leq j \leq s$, or $g^{\prime}=\left(g_{1}\right)^{r}$ and $h_{j}=\left(h_{1}^{j}\right)^{r}$ for every $1 \leq j \leq s$, which is just a proof that one of two sets of tuples are all of the Diffie-Hellman type; see the protocol specification below and the full version for details. The cost of this proof is $s+18$ exponentiations and the exchange of 10 group elements.

\section{PROTOCOL 2 (Single-Choice Cut-and-Choose Oblivious Transfer)}

- Inputs: The sender's input is a vector of s pairs $\left(x_{0}^{j}, x_{1}^{j}\right)$ and the receiver's input is a single bit $\sigma \in\{0,1\}$ and a set $\mathcal{J} \subset[s]$ of size exactly $s / 2$. 
- Auxiliary input: Both parties hold a security parameter $1^{n}$ and $\left(\mathbb{G}, q, g_{0}\right)$, where $\mathbb{G}$ is an efficient representation of a group of order $q$ with a generator $g_{0}$, and $q$ is of length $n$.

\section{- Setup phase:}

1. $R$ chooses a random $y \leftarrow \mathbb{Z}_{q}$ and sets $g_{1}=\left(g_{0}\right)^{y}$.

2. For every $j \in \mathcal{J}, R$ chooses a random $\alpha_{j} \leftarrow \mathbb{Z}_{q}$ and computes $h_{0}^{j}=g_{0}^{\alpha_{j}}$ and $h_{1}^{j}=g_{1}^{\alpha_{j}}$.

3. For every $j \notin \mathcal{J}, R$ chooses random $\alpha_{j} \leftarrow \mathbb{Z}_{q}$ and computes $h_{0}^{j}=g_{0}^{\alpha_{j}}$ and $h_{1}^{j}=g_{1}^{\alpha_{j}+1}$.

4. $R$ sends $\left(g_{1}, h_{0}^{1}, h_{1}^{1}, \ldots, h_{0}^{s}, h_{1}^{s}\right)$ to $S$

5. $R$ proves using a zero-knowledge proof of knowledge to $S$ that $s / 2$ of the tuples $\left(g_{0}, g_{1}, h_{0}^{j}, \frac{h_{1}^{j}}{g_{1}}\right)$ are DH tuples (and through this, that the tuples $\left(g_{0}, g_{1}, h_{0}^{j}, h_{1}^{j}\right)$ are not DH tuples). If $S$ rejects the proof then it outputs $\perp$ and halts.

- Transfer phase (for every $j$ ):

1. The receiver chooses a random value $r \leftarrow \mathbb{Z}_{q}$ and computes $g^{\prime}=\left(g_{\sigma}\right)^{r}$. Then, for every $j$, it computes $h_{j}=\left(h_{\sigma}^{j}\right)^{r}$. It sends $\left(g^{\prime}, h_{1}, \ldots, h_{s}\right)$ to the sender.

2. The receiver proves in zero knowledge that either all $\left\{\left(g_{0}, h_{0}^{j}, g^{\prime}, h_{j}\right)\right\}_{j=1}^{s}$ or all $\left\{\left(g_{1}, h_{1}^{j}, g^{\prime}, h_{j}\right)\right\}_{j=1}^{s}$ are Diffie-Hellman tuples.

3. The sender operates in the following way:

- Define the function $\operatorname{RAND}(w, x, y, z)=(u, v)$, where $u=(w)^{s} \cdot(y)^{t}$ and $v=(x)^{s} \cdot(z)^{t}$, and the values $s, t \leftarrow \mathbb{Z}_{q}$ are random.

- $S$ computes the pairs $\left(u_{0}^{j}, v_{0}^{j}\right)=R A N D\left(g_{0}, g_{j}, h_{0}^{j}, h_{j}\right)$ and $\left(u_{1}^{j}, v_{1}^{j}\right)=$ $R A N D\left(g_{1}, g_{j}, h_{1}^{j}, h_{j}\right)$.

- $S$ sends the receiver the values $\left(u_{0}^{j}, w_{0}^{j}\right)$ where $w_{0}^{j}=v_{0}^{j} \cdot x_{0}^{j}$, and $\left(u_{1}^{j}, w_{1}^{j}\right)$ where $w_{1}^{j}=v_{1}^{j} \cdot x_{1}^{j}$, for every $j$.

\section{- Output:}

1. For every $j \in\{1, \ldots, s\}$, the receiver computes $x_{\sigma}^{j}=w_{\sigma}^{j} /\left(u_{\sigma}^{j}\right)^{r}$.

2. For every $j \in \mathcal{J}$, the receiver also computes $x_{1-\sigma}^{j}=w_{1-\sigma}^{j} /\left(u_{1-\sigma}^{j}\right)^{r \cdot z}$ where $z=y^{-1} \bmod q$ if $\sigma=0$, and $z=y$ if $\sigma=1$.

The fact that the output obtained is correct follows from the correctness (and simulation strategy) of the protocol of [30. We prove the following:

Proposition 3. If the Decisional Diffie-Hellman assumption holds in the group $\mathbb{G}$, then Protocol 2 securely realizes the $\mathcal{F}_{\mathrm{ccot}}^{S}$ functionality in the presence of malicious adversaries.

The proof of Proposition 3 is based on the following ideas. Suppose first that the adversary controls the receiver; we briefly describe an ideal-model simulator "interacting" with the adversary. The simulator receives from the adversary the values $\left(g_{0}, g_{1}\right)$ and $\left(h_{0}^{1}, h_{1}^{1}, \ldots, h_{s}^{0}, h_{s}^{1}\right)$ of the setup phase, and then runs the extractor 
of the zero-knowledge proof of knowledge to learn the witness set. The extracted witnesses identify exactly the set $\mathcal{J}$ of $s / 2$ indices for which $\left(g_{0}, g_{1}, h_{0}^{j}, h_{1}^{j}\right)$ is a Diffie-Hellman tuple, and in addition provides the simulator with the set of values $\alpha_{j}$ for all $j \notin \mathcal{J}$. Next, in the transfer phase, given $g^{\prime}$ and $h_{j}$, the simulator can extract the value of $\sigma$ by simply checking if $\left(g^{\prime}\right)^{\alpha_{j}}=h_{j}$; alternatively, it can run the extractor for the zero-knowledge proof of the transfer phase. The simulator then sends the set $\mathcal{J}$ and bit $\sigma$ to the trusted party and obtains the corresponding outputs. Now, for every $j \in \mathcal{J}$, the simulator received both values $x_{0}^{j}$ and $x_{1}^{j}$ and so can compute $\left(u_{0}^{j}, w_{0}^{j}\right)$ and $\left(u_{1}^{j}, w_{1}^{j}\right)$ just like the honest sender. In contrast, for every $j \notin \mathcal{J}$, the simulator received only $x_{\sigma}^{j}$. In this case, it can still compute $\left(u_{\sigma}^{j}, w_{\sigma}^{j}\right)$ like the honest sender. Regarding $\left(u_{1-\sigma}^{j}, w_{1-\sigma}^{j}\right)$, these can just be taken as uniformly distributed values in $\mathbb{G}$, by the property of the $R A N D$ transformation when applied to non-Diffie-Hellman tuples.

Next consider the case that the adversary controls the sender. In this case, the simulator chooses the $\left(h_{0}^{j}, h_{1}^{j}\right)$ values such that $\left(g_{0}, g_{1}, h_{0}^{j}, h_{1}^{j}\right)$ is a Diffie-Hellman tuple for all $j$. The simulator then "cheats" in the zero-knowledge proof by generating a simulated zero-knowledge proof with the adversary. Given that now all $\left(g_{0}, g_{1}, h_{0}^{j}, h_{1}^{j}\right)$ are Diffie-Hellman tuples, the simulator can extract both inputs $\left(x_{0}^{j}, x_{1}^{j}\right)$ for every $j=1, \ldots, s$, using the same computation as an honest receiver would for $j \in \mathcal{J}$. Finally, the simulator sends the values $\left\{\left(x_{0}^{j}, x_{1}^{j}\right)\right\}_{j=1}^{s}$ to the trusted party, outputs whatever the adversary outputs, and halts. The fact that this is indistinguishable from a real execution follows directly from the indistinguishability of simulated zero-knowledge proofs from real proofs, and from the DDH assumption.

Exact efficiency. In the full version, we present an exact analysis of this protocol, including the cost of all zero-knowledge proofs. The result is that the protocol requires $20.5 s+24$ exponentiations, the exchange of $11 s+15$ group elements, and 6 rounds of communication.

\subsection{Batch Single-Choice Cut-and-Choose OT}

In our protocol we need to carry out cut-and-choose oblivious transfers for all wires in the circuit (where for each wire the input used is $P_{2}$ 's input bit). However, the subset of indices for which the receiver obtains both pairs must be the same in all transfers. This is due to the fact that this determines which of the circuits are checked and which are evaluated, and it is crucial that in all of the evaluated circuits $P_{2}$ only receives one value on every wire. We call a functionality that achieves this "batch single-choice cut-and-choose OT" and denote it $\mathcal{F}_{\text {ccot }}^{S, B}$.

In order to realize this functionality it suffices to run the setup phase of Protocol 2 once (this ensures that the set $\mathcal{J}$ is the same in all executions). Then, the transfer phase of the single-choice protocol is run $\ell$ times in parallel (with the single choice in the $i$ th execution being some $\sigma_{i}$ ). We remark that parallel composition holds here because the simulation only rewinds in the transfer phase for the zero-knowledge protocol, which is zero-knowledge under parallel composition. We have the following: 
Proposition 4. Assuming that the Decisional Diffie-Hellman assumption holds in $\mathbb{G}$, the above-described protocol securely realizes $\mathcal{F}_{\mathrm{ccot}}^{S, B}$ in the presence of $m a-$ licious adversaries.

Exact efficiency. The setup phase here remains the same, and including the zero-knowledge proof it costs $9 s+5$ exponentiations and the exchange of $5 s+5$ group elements. The transfer phase is repeated $\ell$ times, where each transfer incurs a cost of $11.5 s+19$ exponentiations and the exchange of $6 s+10$ group elements. We conclude that there are $\mathbf{1 1 . 5} \boldsymbol{s} \boldsymbol{\ell}+\mathbf{1 9} \boldsymbol{\ell}+\mathbf{9} s+\mathbf{5}$ exponentiations, $6 s \ell+10 \ell+5 s+5$ group elements sent and 6 rounds of communication. In Section 3.3 we observe that $10.5 s \ell$ of the exponentiations are "fixed-base" and thus the overall effective cost is about $4.5 s \ell$ exponentiations.

\section{The Protocol for Secure Two-Party Computation}

\subsection{Protocol Description}

Before describing the protocol in detail, we first present an intuitive explanation of the different steps, and their purpose:

Step 1: $P_{1}$ constructs $s$ copies of a Yao garbled circuit for computing the function. The keys (garbled values) on the wires of the $s$ copies of the circuit are all random, except for the keys corresponding to $P_{1}$ 's input wires, which are chosen in a special way. Namely, $P_{1}$ chooses random values $a_{1}^{0}, a_{1}^{1}, \ldots, a_{\ell}^{0}, a_{\ell}^{1}$ (where the length of $P_{1}$ 's input is $\ell$ ) and $r_{1}, \ldots, r_{s}$, and sets the keys on the wire associated with its $i$ th input in the $j$ th circuit to be $g^{a_{i}^{0} \cdot r_{j}}$ and $g^{a_{i}^{1} \cdot r_{j}}$. Note that the $2 \ell+s$ values $g^{a_{1}^{0}}, g^{a_{1}^{1}}, \ldots, g^{a_{\ell}^{0}}, g^{a_{\ell}^{1}}, g^{r_{1}}, \ldots, g^{r_{s}}$ constitute commitments to all $2 \ell s$ keys. (The keys are actually a pseudorandom synthesizer [27, and therefore if some of the keys are revealed, the remaining keys remain pseudorandom).

Step 2: The parties execute batch single-choice cut-and-choose OT. $P_{1}$ inputs the key-pairs for all wires associated with $P_{2}$ 's input, and $P_{2}$ inputs its input and a random set $\mathcal{J} \subset[s]$ of size $s / 2$. The result is that $P_{2}$ learns all the keys on the wires associated with its own input for $s / 2$ of the circuits as indexed by $\mathcal{J}$ (called check circuits), and in addition learns the keys corresponding to its actual input in these wires in the remaining circuits (called evaluation circuits).

Step 3: $P_{1}$ sends $P_{2}$ the garbled circuits, and the values $g^{a_{1}^{0}}, g^{a_{1}^{1}}, \ldots, g^{a_{\ell}^{0}}, g^{a_{\ell}^{1}}$, $g^{r_{1}}, \ldots, g^{r_{s}}$ which are commitments to all the keys on the wires associated with $P_{1}$ 's input. Observe that at this stage $P_{1}$ is fully committed to all $s$ circuits, but does not yet know which circuits are to be opened.

${ }^{1}$ The actual symmetric keys used are derived from the $g^{a_{i}^{0} \cdot r_{j}}, g^{a_{i}^{1} \cdot r_{j}}$ values using a randomness extractor; a universal hash function suffices for this 6 616. The only subtlety is that $P_{1}$ must be fully committed to the garbled circuits, including these symmetric keys, before it knows which circuits are to be checked. However, randomness extractors are not $1-1$ functions. This is solved by having $P_{1}$ send the seed for the extractor before Step 4 below. Observe that the $\left\{g^{a_{i}^{0}}, g^{a_{i}^{1}}, g^{r_{j}}\right\}$ values and the seed for the extractor fully determine the symmetric keys, as required. 
Step 4: $P_{2}$ reveals to $P_{1}$ its choice of check circuits and proves that was indeed its choice by sending, for each check circuit, both values on the wire associated with $P_{2}$ 's first input bit. Note that $P_{2}$ can know both these values only for circuits that are check circuits.

Step 5: To completely decrypt the check circuits in order to check that they were correctly constructed, $P_{2}$ also needs to obtain all the keys on the wires associated with $P_{1}$ 's input. Therefore, if the $j$ th circuit is a check circuit, then $P_{1}$ sends $r_{j}$ to $P_{2}$. Given all of the $g^{a_{i}^{0}}, g^{a_{i}^{1}}$ values and $r_{j}$, party $P_{2}$ can compute all of the keys $g^{a_{i}^{0} \cdot r_{j}}, g^{a_{i}^{1} \cdot r_{j}}$ in the $j$ th circuit by itself (and $P_{1}$ cannot change the values). Furthermore, this reveals nothing about the keys in the evaluation circuits.

Step 6: Given all of the keys on all of the input wires, $P_{2}$ checks the validity of the $s / 2$ check circuits. This ensures that $P_{2}$ will catch $P_{1}$ with high probability if many of the garbled circuits generated by $P_{1}$ do not compute the correct function. Thus, unless $P_{2}$ detects cheating, it is assured that a majority of the evaluation circuits are correct.

Step 7: All that remains is for $P_{1}$ to send $P_{2}$ the keys associated with its actual input, and then $P_{2}$ will be able to compute the evaluation circuits. This raises a problem as to how $P_{2}$ can be sure that $P_{1}$ sends keys that correspond to the same input in all circuits. This brings us to the way that $P_{1}$ chose these keys (via the Diffie-Hellman pseudorandom synthesizer). Specifically, for every wire $i$ and evaluation-circuit $j$, party $P_{1}$ sends $P_{2}$ the value $g^{a_{i}^{x_{i}}} \cdot r_{j}$ where $x_{i}$ is the $i$ th bit of $P_{1}$ 's input. $P_{1}$ then proves in zero-knowledge that the same $a_{i}^{x_{i}}$ exponent appears in all of the values sent. Essentially, this is a proof that the values constitute an "extended" Diffie-Hellman tuple and thus this statement can be proven very efficiently.

Step 8: Finally, given the keys associated with $P_{1}$ 's inputs and its own inputs, $P_{2}$ evaluates the evaluation circuits and obtains their output values. Recall, however, that the checks above only guarantee that a majority of the circuits are correct, and not that all of them are. Therefore, $P_{2}$ outputs the value that is output from the majority of the evaluation circuits. We stress that if $P_{2}$ sees different outputs in different circuits, and thus knows for certain that $P_{1}$ has tried to cheat, it must ignore this observation and output the majority value (or otherwise it might leak information to $P_{1}$, as in the example described in Section 1.2).

We remark on one type of attack discussed in 2124]. The concern there was that $P_{1}$ would use correct keys for all of $P_{2}$ 's input bits when opening the check circuit, but would use incorrect keys in some of the oblivious transfers. This is problematic because if $P_{1}$ input incorrect keys for the zero value of $P_{2}$ 's first input bit, and correct keys for all other values, then $P_{2}$ would not detect any misbehavior if its first input bit equals 1 . However, if its first input bit equals 0 then it would have to abort (because it would not be able to decrypt any of the evaluation circuits). This results in $P_{1}$ learning $P_{2}$ 's first input bit with probability 1. In order to solve this problem in 24] it was necessary to split $P_{2}$ 's input bits into random shares, thereby increasing the size of the input to 
the circuit and the size of the circuit itself. In contrast, this attack does not arise here at all because $P_{2}$ obtains all of the keys associated with its input bits in the cut-and-choose oblivious transfer, and the values are not sent separately for check and evaluation circuits. Thus, if $P_{1}$ attempts a similar attack here for a small number of circuits then it will not be the majority and so does not matter, and if it does so for a large number of circuits then it will be caught with overwhelming probability. We now proceed to the full protocol description.

\section{PROTOCOL 5 (Computing $f(x, y)$ )}

Inputs: $P_{1}$ has input $x \in\{0,1\}^{\ell}$ and $P_{2}$ has input $y \in\{0,1\}^{\ell}$.

Auxiliary input: a statistical security parameter $s$, the description of a circuit $C$ such that $C(x, y)=f(x, y)$, and $(\mathbb{G}, q, g)$ where $\mathbb{G}$ is a cyclic group with generator $g$ and prime order $q$, and $q$ is of length $n$.

\section{The protocol:}

1. INPUT KEY CHOICE AND CIRCUIT PREPARATION:

(a) $P_{1}$ chooses random values $a_{1}^{0}, a_{1}^{1}, \ldots, a_{\ell}^{0}, a_{\ell}^{1} \in \in_{R} \mathbb{Z}_{q}$ and $r_{1}, \ldots, r_{s} \in_{R} \mathbb{Z}_{q}$.

(b) Let $w_{1}, \ldots, w_{\ell}$ be the input wires corresponding to $P_{1}$ 's input in $C$, and denote by $w_{i, j}$ the instance of wire $w_{i}$ in the $j$ th garbled circuit, and by $k_{i, j}^{b}$ the key associated with bit $b$ on wire $w_{i, j}$. Then, $P_{1}$ sets the keys for its input wires to: $k_{i, j}^{0}=H\left(g^{a_{i}^{0} \cdot r_{j}}\right)$ and $k_{i, j}^{1}=H\left(g^{a_{i}^{1} \cdot r_{j}}\right)$, where $H$ is a suitable randomness extractor [6 16]; see also [10].

(c) $P_{1}$ constructs $s$ independent copies of a garbled circuit of $C$, denoted $G C_{1}, \ldots, G C_{s}$, using random keys except for wires $w_{1}, \ldots, w_{\ell}$ for which the keys are as above.

2. Oblivious transfers: $P_{1}$ and $P_{2}$ run batch single-choice cut-and-choose oblivious transfer with parameters $\ell$ (the number of parallel executions) and $s$ (the number of pairs in each execution):

(a) $P_{1}$ defines vectors $\boldsymbol{z}_{1}, \ldots \boldsymbol{z}_{\ell}$ so that $\boldsymbol{z}_{i}$ contains the $s$ pairs of random symmetric keys associated with $P_{2}$ 's ith input bit $y_{i}$ in all garbled circuits $G C_{1}, \ldots, G C_{s}$.

(b) $P_{2}$ inputs a random subset $\mathcal{J} \subset[s]$ of size exactly $s / 2$ and bits $\sigma_{1}, \ldots$, $\sigma_{\ell} \in\{0,1\}$, where $\sigma_{i}=y_{i}$ for every $i$.

(c) $P_{2}$ receives all the keys associated with its input wires in all circuits $G_{j}$ for $j \in \mathcal{J}$, and receives the keys associated with its input $y$ on its input wires in all other circuits.

3. Send CiRCuits And COMmitments: $P_{1}$ sends $P_{2}$ the garbled circuits (i.e., the gate and output tables), the "seed" for the randomness extractor $H$, and the following "commitment" to the garbled values associated with $P_{1}$ 's input wires: $\left\{\left(i, 0, g^{a_{i}^{0}}\right),\left(i, 1, g^{a_{i}^{1}}\right)\right\}_{i=1}^{\ell}$ and $\left\{\left(j, g^{r_{j}}\right)\right\}_{j=1}^{s}$.

4. Send Cut-And-Choose Challenge: $P_{2}$ sends $P_{1}$ the set $\mathcal{J}$ along with the pair of keys associated with its first input bit $y_{1}$ in every circuit $G C_{j}$ for $j \in \mathcal{J}$. If the values received by $P_{1}$ are incorrect, it outputs $\perp$ and aborts. Circuits $G C_{j}$ for $j \in \mathcal{J}$ are called check-circuits, and for $j \notin \mathcal{J}$ are called evaluation-circuits. 
5. Send All inPut Garbled values in CHECK-CIRCuIts: For every checkcircuit $G C_{j}$, party $P_{1}$ sends the value $r_{j}$ to $P_{2}$, and $P_{2}$ checks that these are consistent with the pairs $\left\{\left(j, g^{r_{j}}\right)\right\}_{j \in \mathcal{J}}$ received in Step [3. If not, $P_{2}$ aborts outputting $\perp$.

6. Correctness of CHECK CiRCuits: For every $j \in \mathcal{J}, P_{2}$ uses the $g^{a_{i}^{0}}, g^{a_{i}^{1}}$ values it received in Step [3, and the $r_{j}$ values it received in Step 5, to compute the values $k_{i, j}^{0}=H\left(g^{a_{i}^{0} \cdot r_{j}}\right), k_{i, j}^{1}=H\left(g^{a_{i}^{1} \cdot r_{j}}\right)$ associated with $P_{1}$ 's input in $G C_{j}$. In addition it sets the garbled values associated with its own input in $G C_{j}$ to be as obtained in the cut-and-choose OT. Given all the garbled values for all input wires in $G C_{j}$, party $P_{2}$ decrypts the circuit and verifies that it is a garbled version of $C$.

7. $P_{1}$ SEnds its GARBled inPut VAlues in THE EVAluAtion-CIRCUITS:

(a) $P_{1}$ sends the keys associated with its inputs in the evaluation circuits: For every $j \notin \mathcal{J}$ and every wire $i=1, \ldots, \ell$, party $P_{1}$ sends the value $k_{i, j}^{\prime}=g^{a_{i}^{x_{i}} \cdot r_{j}} ; P_{2}$ sets $k_{i, j}=H\left(k_{i, j}^{\prime}\right)$.

(b) $P_{1}$ proves that all input values are consistent: For every input wire $i=$ $1, \ldots, \ell$, party $P_{1}$ proves in parallel that there exists a value $\sigma_{i} \in\{0,1\}$ such that for every $j \notin J, k_{i, j}^{\prime}=g^{a_{i}^{\sigma_{i}} \cdot r_{j}}$. If any of the proofs fail, then $P_{2}$ aborts and outputs $\perp$.

8. Circuit evaluation: $P_{2}$ uses the keys associated with $P_{1}$ 's input obtained in Step $7 a$ and the keys associated with its own input obtained in Step $2 \mathrm{C}$ to evaluate the evaluation circuits $G C_{j}$ for every $j \notin \mathcal{J}$. If a circuit decryption fails, then $P_{2}$ sets the output of that circuit to be $\perp$. Party $P_{2}$ takes the output that appears in most circuits, and outputs it.

\subsection{Properties}

The security of the protocol is expressed in the following theorem, which is proved in the full version of the paper:

Theorem 6. Assume that the decisional Diffie-Hellman assumption is hard in $\mathbb{G}$, that the protocol used in Step 2 securely computes the batch single-choice cutand-choose oblivious transfer functionality, that the protocol used in Step 76 is a zero-knowledge proof of knowledge, and that the symmetric encryption scheme used to generate the garbled circuits is secure. Then, Protocol 5 securely computes the function $f$ in the presence of malicious adversaries.

We remark here on one aspect of the proof that is crucial to the concrete efficiency of the protocol. Party $P_{1}$ can successfully cheat if it manages to pass the cut-andchoose test with a majority of the evaluation circuits being incorrect. To do this, at least $s / 4$ circuits must be incorrect. In the proof of security we show that the probability that at least this many circuits are incorrect without $P_{2}$ catching $P_{1}$ is approximately $2^{-0.311 s}$ where the approximation is due to Stirling's formula. Based on this, it suffices to use 128 garbled circuits in order to obtain an error of $2^{-40}$. (We also compared the exact bound to this approximation on the concrete value of $s=128$, to verify that the approximation is good for $s$ of this size). 
Exact efficiency. An exact analysis of the protocol yields that there are $\mathbf{1 5} \boldsymbol{s} \boldsymbol{\ell +}$ $\mathbf{3 9} \ell+\mathbf{1 0 s}+\mathbf{5}$ exponentiations (of which 11.5s $\ell$ are for Step 2, performing the OTs), $7 s \ell+22 \ell+7 s+5$ group elements sent and $\mathbf{1 2}$ rounds of communication. In addition, there are $6.5|C| s$ symmetric encryptions, of which $4|C| s$ encryptions for constructing all $s$ garbled circuits, and $2|C| s$ encryptions for $P_{2}$ to check $s / 2$ of them. Finally, there are $4|C| s$ ciphertexts sent for transmitting these circuits. The overhead of the protocol can be improved by different optimizations, as shown in Section 3.3 below.

\subsection{Optimizations}

Fixed-base exponentiations. Exponentiations are commonly computed by repeated squaring, which for a group of order $q$ of length $m$ bits requires on average $1.5 \mathrm{~m}$ multiplications for a full exponentiation. If multiple exponentiations of the same base are computed, then the repeated binary powers of the base can be computed once for all exponentiations, reducing the amortized overhead of an exponentiation on average to $0.5 \mathrm{~m}$ multiplications. All but $s \ell$ of the exponentiations in our protocol are fixed-based, and thus taking this into account the effective overhead of the exponentiations is equivalent to that of just $\mathbf{5 . 6 6 s \boldsymbol { s }}$ full exponentiations.

Reducing the computation of $P_{2}$ in Step 6. In Step 6] of Protocol 5, $P_{2}$ performs $s \ell$ exponentiations in order to compute the garbled values associated with $P_{1}$ 's input in the check circuits. Namely, given the $\left(i, 0, g^{a_{i}^{0}}\right),\left(i, 1, g^{a_{i}^{1}}\right)$ tuples and $r_{j}$ for every $j \in \mathcal{J}$, party $P_{2}$ computes $g^{a_{i}^{0} \cdot r_{j}}, g^{a_{i}^{1} \cdot r_{j}}$ for all $i=1 \ldots \ell$ and $j \in \mathcal{J}$. This step costs $s \ell$ exponentiations $(2 \ell$ exponentiations for each of the $s / 2$ check circuits). We can reduce this to about a quarter by having $P_{1}$ send the $g^{a_{i}^{0} \cdot r_{j}}, g^{a_{i}^{1} \cdot r_{j}}$ values to $P_{2}$ and prove that they are correct (not in zero-knowledge).

The protocol is modified by changing Step 6 as follows (recall that $P_{2}$ already has all of the $\left(i, 0, g^{a_{i}^{0}}\right),\left(i, 1, g^{a_{i}^{1}}\right)$ tuples and $r_{j}$ values $)$ :

1. $P_{1}$ sends $P_{2}$ all of the values ${k^{\prime}}_{i, j}^{0}=g^{a_{i}^{0} \cdot r_{j}}$ and $k_{i, j}^{\prime 1}=g^{a_{i}^{1} \cdot r_{j}}$ for $i=1, \ldots, \ell$ and $j \in \mathcal{J}$.

2. $P_{2}$ chooses random values $\gamma_{i}^{0}, \gamma_{i}^{1} \in\left[1,2^{L}\right]$ for $i=1, \ldots, \ell$.

3. For every $j \in \mathcal{J}$, party $P_{2}$ computes the values $\alpha_{j}=\left(\prod_{i=1}^{\ell}\left(g^{a_{i}^{0}}\right)^{\gamma_{i}^{0}} \cdot\left(g^{a_{i}^{1}}\right)^{\gamma_{i}^{1}}\right)^{r_{j}}$ and $\beta_{j}=\prod_{i=1}^{\ell}\left({k^{\prime}}_{i, j}^{0}\right)^{\gamma_{i}^{0}} \cdot\left({k^{\prime}}_{i, j}^{1}\right)^{\gamma_{i}^{1}}$ Note that computing $\alpha_{j}$ requires only a single full exponentiation since the value $\left(g^{a_{i}^{0}}\right)^{\gamma_{i}^{0}} \cdot\left(g^{a_{i}^{1}}\right) \gamma_{i}^{1}$ can be computed once for all $j$.

4. $P_{2}$ accepts $P_{1}$ 's input if and only if $\alpha_{j}=\beta_{j}$ for all $j \in \mathcal{J}$.

Claim 7. The probability that $P_{2}$ accepts if there exists an $i \in\{1, \ldots, \ell\}$ and $j \in \mathcal{J}$ such that ${k^{\prime}}_{i, j}^{0} \neq g^{a_{i}^{0} \cdot r_{j}}$ or ${k^{\prime}}_{i, j}^{1} \neq g^{a_{i}^{1} \cdot r_{j}}$ is at most $\frac{s}{2} \cdot 2^{-L}$.

Preprocessing. The bulk of the exponentiations performed in the protocol can be precomputed. Step 1 of the protocol, where $P_{1}$ computes its input keys, can clearly be computed before $P_{1}$ receives its inputs. Step 2 executes the oblivious 
transfers. It can be slightly changed to be run before $P_{2}$ receives its inputs: $P_{2}$ can execute this step with random inputs $\sigma_{1}, \ldots, \sigma_{\ell}$. Then, when it receives its input bits $y_{1}, \ldots, y_{\ell}$, it sends to $P_{1}$ a string of correction bits $y_{1} \oplus \sigma_{1}, \ldots, y_{\ell} \oplus \sigma_{\ell}$. $P_{1}$ exchanges the roles of the two keys of input wires of $P_{2}$ for which it receives a correction bit with the value 1 . (The security proof can be easily adapted for this variant of the protocol). Given this change, both Steps 1 and 2 can be precomputed. These steps account for $13.5 \mathrm{~s} \ell$ of the $15 \mathrm{~s} \ell$ exponentiations of the protocol, where the remaining $1.5 \mathrm{~s} \ell$ exponentiations are fixed base. This means that if preprocessing is used, then after receiving their inputs the parties need to effectively compute only $s \ell / 2$ full exponentiations.

\section{Universal Composability and Covert Adversaries}

\subsection{Universally Composable Two-Party Computation}

The simulators in the proof of Theorem 6 carry out no rewinding, and likewise the intermediate simulators used to prove the reductions. Thus, if the protocols used to compute the batch cut-and-choose oblivious transfer functionality and the zero-knowledge proof of knowledge of Step $7 \mathrm{~b}$ are universally composable, then so is Protocol 5. In order to obtain this property, we simply need to apply a transformation from Sigma protocols to universally-composable zero knowledge, which can be achieved efficiently using universally-composable commitments. Details of how this can be achieved are given in the full version of the paper. We have the following:

Theorem 8. Assume that the decision Diffie-Hellman assumption holds. Then, for every efficiently computable two-party function $f$ with inputs of length $\ell$, there exists a universally composable protocol that securely computes $f$ in the commitment-hybrid model in the presence of malicious adversaries, with 8 rounds of computation and $O\left(s \ell+s^{2}\right)$ exponentiations.

\subsection{Covert Security}

In the model of security in the presence of covert adversaries [1], the requirement is that any cheating by an adversary will be caught with some probability $\epsilon$. The value of $\epsilon$ taken depends on the application, the ramifications to an adversary being caught, the value to an adversary of successfully cheating (if not caught) and so on. The analysis of our protocol shows that for every value of $s$ (even if $s$ is very small) the probability that an adversary can cheat without being caught is at most $2^{-\frac{s}{4}+1}$. This immediately yields a protocol that is secure in the presence of covert adversaries, as stated in the following theorem.

Theorem 9. Assume that the decisional Diffie-Hellman assumption is hard in $\mathbb{G}$, that the protocol used in Step 2 securely computes the batch single-choice cutand-choose oblivious transfer functionality, that the protocol used in Step $7 b$ is a zero-knowledge proof of knowledge, and that the symmetric encryption scheme 
used to generate the garbled circuit is secure. Then, for any integer $s>4$, Protocol 55 securely computes the function $f$ in the presence of covert adversaries with $\epsilon$-deterrent (under the strong explicit cheat formulation), for $\epsilon=1-2^{-\frac{s}{4}+1}$.

We stress that our protocol is significantly more efficient than the protocols of [1] and 15. when values of $\epsilon$ that are greater than $1 / 2$ are desired. For example, in order to obtain an $\epsilon$-deterrent of 0.98 , the protocol of [1] requires using 50 garbled circuits. However, taking $s=50$ in our protocol here yields an $\epsilon$-deterrent of $1-2^{-11.5}$ which is much much larger.

\section{Acknowledgements}

We thank Bo Zhang for pointing out an error in the single-choice cut-and-choose oblivious transfer protocol in an earlier version of this work.

\section{References}

1. Aumann, Y., Lindell, Y.: Security Against Covert Adversaries: Efficient Protocols for Realistic Adversaries. Journal of Cryptology 23(2), 281-343 (2010)

2. Beaver, D.: Foundations of Secure Interactive Computing. In: Feigenbaum, J. (ed.) CRYPTO 1991. LNCS, vol. 576, pp. 377-391. Springer, Heidelberg (1992)

3. Canetti, R.: Security and Composition of Multi-party Cryptographic Protocols. Journal of Cryptology 13(1), 143-202 (2000)

4. Canetti, R.: Universally Composable Security: A New Paradigm for Cryptographic Protocols. In: 42nd FOCS, pp. 136-145 (2001), Full version http://eprint.iacr.org/2000/067

5. Canetti, R., Fischlin, M.: Universally Composable Commitments. In: Kilian, J. (ed.) CRYPTO 2001. LNCS, vol. 2139, pp. 19-40. Springer, Heidelberg (2001)

6. Carter, L., Wegman, M.N.: Universal Classes of Hash Functions. JCSS 18(2), 143$154(1979)$

7. Cramer, R., Damgård, I., Schoenmakers, B.: Proof of partial knowledge and simplified design of witness hiding protocols. In: Desmedt, Y.G. (ed.) CRYPTO 1994. LNCS, vol. 839, pp. 174-187. Springer, Heidelberg (1994)

8. Damgård, I.: On $\Sigma$ Protocols, http://www.daimi.au.dk/ ivan/Sigma.pdf

9. Dodis, Y., Shoup, V., Walfish, S.: Efficient Constructions of Composable Commitments and Zero-Knowledge Proofs. In: Wagner, D. (ed.) CRYPTO 2008. LNCS, vol. 5157, pp. 515-535. Springer, Heidelberg (2008)

10. Dodis, Y., Gennaro, R., Håstad, J., Krawczyk, H., Rabin, T.: Randomness Extraction and Key Derivation Using the CBC, Cascade and HMAC Modes. In: Franklin, M. (ed.) CRYPTO 2004. LNCS, vol. 3152, pp. 494-510. Springer, Heidelberg (2004)

11. Garay, J., MacKenzie, P., Yang, K.: Strengthening Zero-Knowledge Protocols Using Signatures. In: Biham, E. (ed.) EUROCRYPT 2003. LNCS, vol. 2656, pp. 177-194. Springer, Heidelberg (2003)

12. Goldreich, O.: Foundations of Cryptography: Volume 2 - Basic Applications. Cambridge University Press, Cambridge (2004)

13. Goldreich, O., Micali, S., Wigderson, A.: How to Play any Mental Game - A Completeness Theorem for Protocols with Honest Majority. In: 19th STOC, pp. 218-229 (1987), For details see [12, Chapter 7] 
14. Goldwasser, S., Levin, L.: Fair Computation of General Functions in Presence of Immoral Majority. In: Menezes, A., Vanstone, S.A. (eds.) CRYPTO 1990. LNCS, vol. 537, pp. 77-93. Springer, Heidelberg (1991)

15. Goyal, V., Mohassel, P., Smith, A.: Efficient Two Party and Multi Party Computation Against Covert Adversaries. In: Smart, N.P. (ed.) EUROCRYPT 2008. LNCS, vol. 4965, pp. 289-306. Springer, Heidelberg (2008)

16. Hastad, J., Impagliazzo, R., Levin, L., Luby, M.: Construction of a Pseudo-random Generator from any One-way Function. SIAM Journal on Computing 28(4), 13641396 (1999)

17. Hazay, C., Nissim, K.: Efficient Set Operations in the Presence of Malicious Adversaries. In: Nguyen, P.Q., Pointcheval, D. (eds.) PKC 2010. LNCS, vol. 6056, pp. 312-331. Springer, Heidelberg (2010)

18. Ishai, Y., Prabhakaran, M., Sahai, A.: Founding Cryptography on Oblivious Transfer - Efficiently. In: Wagner, D. (ed.) CRYPTO 2008. LNCS, vol. 5157, pp. 572-591. Springer, Heidelberg (2008)

19. Ishai, Y., Prabhakaran, M., Sahai, A.: Secure Arithmetic Computation with No Honest Majority. In: Reingold, O. (ed.) TCC 2009. LNCS, vol. 5444, pp. 294-314. Springer, Heidelberg (2009)

20. Jarecki, S., Shmatikov, V.: Efficient Two-Party Secure Computation on Committed Inputs. In: Naor, M. (ed.) EUROCRYPT 2007. LNCS, vol. 4515, pp. 97-114. Springer, Heidelberg (2007)

21. Kiraz, M., Schoenmakers, B.: A Protocol Issue for the Malicious Case of Yao's Garbled Circuit Construction. In: Proceedings of 27th Symposium on Information Theory in the Benelux, pp. 283-290 (2006)

22. Lindell, Y.: Parallel Coin-Tossing and Constant-Round Secure Two-Party Computation. Journal of Cryptology 16(3), 143-184 (2003)

23. Lindell, Y., Pinkas, B.: A Proof of Yao's Protocol for Secure Two-Party Computation. The Journal of Cryptology 22(2), 161-188 (2009)

24. Lindell, Y., Pinkas, B.: An Efficient Protocol for Secure Two-Party Computation in the Presence of Malicious Adversaries. In: Naor, M. (ed.) EUROCRYPT 2007. LNCS, vol. 4515, pp. 52-78. Springer, Heidelberg (2007)

25. MacKenzie, P., Yang, K.: On Simulation-Sound Trapdoor Commitments. In: Cachin, C., Camenisch, J.L. (eds.) EUROCRYPT 2004. LNCS, vol. 3027, pp. 382400. Springer, Heidelberg (2004)

26. Micali, S., Rogaway, P.: Secure Computation. In: Feigenbaum, J. (ed.) CRYPTO 1991. LNCS, vol. 576, pp. 392-404. Springer, Heidelberg (1992)

27. Naor, M., Reingold, O.: Synthesizers and Their Application to the Parallel Construction of Psuedo-Random Functions. In: 36th FOCS, pp. 170-181 (1995)

28. Nielsen, J.B., Orlandi, C.: LEGO for Two-Party Secure Computation. In: Reingold, O. (ed.) TCC 2009. LNCS, vol. 5444, pp. 368-386. Springer, Heidelberg (2009)

29. Orlandi, C.: Personal communication (2010)

30. Peikert, C., Vaikuntanathan, V., Waters, B.: A Framework for Efficient and Composable Oblivious Transfer. In: Wagner, D. (ed.) CRYPTO 2008. LNCS, vol. 5157, pp. 554-571. Springer, Heidelberg (2008)

31. Pinkas, B., Schneider, T., Smart, N.P., Williams, S.C.: Secure Two-Party Computation Is Practical. In: Matsui, M. (ed.) ASIACRYPT 2009. LNCS, vol. 5912, pp. 250-267. Springer, Heidelberg (2009)

32. Shamir, A.: How to Share a Secret. Communications of the ACM 22(11), 612-613 (1979)

33. Yao, A.C.: How to Generate and Exchange Secrets. In: 27th FOCS, pp. 162-167 (1986) 